\title{
The study protocol for a pseudo-randomised pre-post designed controlled intervention trial to study the effects of a 7-week cooking program on self-efficacy and biomarkers of health: the ECU lifestyle and biomarkers get connected study (ECULABJMOF) including the Jamie's Ministry of Food WA participant experience
}

Joanna Rees ${ }^{1 *}$ (D), Claus C. Christophersen ${ }^{1,2}$, Joshua R. Lewis ${ }^{1,3,4}$, Johnny Lo ${ }^{5}$, Ros Sambell ${ }^{1}$, Leesa Costello ${ }^{1}$, Cailyn Walker ${ }^{1}$, Matt F. Byrne ${ }^{6}$, Mary C. Boyce ${ }^{5,7}$, Robert U. Newton ${ }^{1,8,9}$ and Amanda Devine ${ }^{1}$

\begin{abstract}
Background: Australia, like other nations, has experienced a shift in dietary patterns away from home cooking of nutritious foods, towards a reliance on pre-prepared convenience meals. These are typically energy-dense, nutrientpoor and contribute to the rising prevalence of obesity and chronic disease burden. The aims of this study were to evaluate whether a community-based cooking program instigated a change to participants' skills, attitudes, knowledge, enjoyment and satisfaction of cooking and cooking confidence (self-efficacy).

Methods: The pseudo-random, pre-post study design consisted of an intervention and a control group. Participant recruitment and group allocation was based on their program start dates. Intervention participants were surveyed three times (baseline, 7 weeks and 6 months) and the control group were surveyed at baseline and 5 weeks. All participants were registered via an online website and were 18 years or over. Upon consent, participants were offered four levels of commitment, defined by different assessments. The minimum participation level included an online survey and levels 2, 3 and 4 involved attendance at a clinic with increasing functional, anthropometric and biomarker measurements. Primary endpoints were participants' cooking confidence as a proxy for self-efficacy. Secondary endpoints were dietary intake, physical activity levels, body composition, anthropometry, blood, urine and faecal biomarkers of systemic, physical and mental health.

(Continued on next page)
\end{abstract}

* Correspondence: j.rees@ecu.edu.au

'School of Medical and Health Sciences, Edith Cowan University, 270

Joondalup Drive, Joondalup, Perth, WA 6027, Australia

Full list of author information is available at the end of the article

() The Author(s). 2020 Open Access This article is licensed under a Creative Commons Attribution 4.0 International License, which permits use, sharing, adaptation, distribution and reproduction in any medium or format, as long as you give appropriate credit to the original author(s) and the source, provide a link to the Creative Commons licence, and indicate if changes were made. The images or other third party material in this article are included in the article's Creative Commons licence, unless indicated otherwise in a credit line to the material. If material is not included in the article's Creative Commons licence and your intended use is not permitted by statutory regulation or exceeds the permitted use, you will need to obtain permission directly from the copyright holder. To view a copy of this licence, visit http://creativecommons.org/licenses/by/4.0/. The Creative Commons Public Domain Dedication waiver (http://creativecommons.org/publicdomain/zero/1.0/) applies to the data made available in this article, unless otherwise stated in a credit line to the data. 
(Continued from previous page)

Discussion: The community cooking program provided participants with information and advice on food sourcing, preparation and nutrition to improve home cooking skills. The study was designed to explore whether food literacy programs are efficacious in improving participant physical health and well-being in order to combat the rise in obesity and diet-related disease. It will support future use of public health cooking program initiatives aimed at improving food literacy, self-efficacy and physical and mental health. The extensive data collected will inform future research into the relationship between diet, the gutmicrobiota and human health.

Trial registration: Retrospectively registered on 16.08.2019 with the Australian New Zealand Clinical Trials Registry (ANZCTR). ACTRN12619001144101.

Protocol version 4.

Keywords: Cooking program, Self-efficacy, Dietary intake, Microbiota, Mental health, Study protocol

\section{Background}

Healthy eating patterns and good food choices are essential for promoting health and well-being and preventing a wide range of chronic diseases [1], such as cardiovascular disease [2], Type 2 diabetes [3], some cancers [4] and obesity [5]. Yet over the past few decades, Australia like most other nations has experienced a dramatic shift in daily dietary patterns away from preparing and cooking nutritious home food, towards a significant reliance on pre-packaged, prepared, convenience meals [6, 7]. This shift is reflected by current statistics showing that those with insufficient fruit and vegetable intake has risen by $8.5 \%$ for men and $10.8 \%$ for women since $2004 / 5$ [8]. The increasing demands of today's modern lifestyles appear to limit the amount of time spent on home cooking. In response, the food industry has grown substantially, offering fast food and ready-made meals which are often energy-dense, nutrient-poor, with high levels of salt, saturated fat and sugar [9].

The Australian Bureau of Statistics (ABS) National Health Survey (2017-18) [10] showed $67.0 \%$ of Australian adults were overweight or obese (12.5 million people), an increase from $63.4 \%$ in 2014 , placing a considerable burden on the healthcare budget and economic growth [11]. In addition, mental health and behavioural conditions affect $20 \%$ of the Australian population [10]. Epidemiological studies have found that depressive disorders are closely linked to cardiovascular and metabolic health outcomes and share a similar aetiology [12-14]. There is overwhelming evidence that a transition towards a Westernised diet high in processed foods, fats and protein and low in fresh fruit, vegetables and dietary fibre (DF) is associated with higher rates of both metabolic disease and mental health disorders [15-17]. Further the effect of a Western diet on the gut microbiome has been reported to induce dysbiosis, that is related to a compromised metabolic profile in the gut, which impacts mental and physiological health $[18,19]$.
Social determinants of health such as socio-economic status and household income are associated with food purchasing decisions and dietary intake, whereby households of lower income and those in rural and remote areas often have poor diet quality and greater risk of disease [20-22]. Barriers such as the price of fresh produce and lack of cooking skills can influence purchasing behaviours for healthier foods, especially among lower socio-economic families and those who are food insecure [23-25]. Therefore providing individuals, families and communities with food literacy and access to information to develop budgeting skills, efficient food shopping strategies, and skills to prepare healthy meals at low cost, may overcome economic barriers that presently prevent healthy eating $[26,27]$. The proliferation of community-based cooking interventions to address these issues is well documented [23, 24, 28-32] and interest has been stimulated by media attention and prime time cooking programs with celebrity chefs [33]. However, to date the evidence of efficacy has been based on small-scale evaluations with methodological limitations $[24,34]$. During the lead-up to this study, various systematic reviews of community cooking interventions reported that, although most observed positive changes in cooking confidence, findings were inconclusive due to a lack of quality evaluation methods and small convenience samples with female predominance [35-37]. A later systematic review [30], (published after the commencement of this study), further emphasised the need for future intervention studies to include a control group, follow-up beyond program completion and the use of validated assessment instruments. Reicks et al. [30] also reported that there was still a limited number of studies that included clinical measures of health and lifestyle.

These reviews emphasise the need for more rigorous, large-scale longitudinal studies to examine the range of impacts and outcomes of cooking skill programs and underlying factors that influence behavioural change [34] and that include potential drivers that are influenced by health, taste, cost, time, convenience, family responsibilities, familiarity and confidence [38-41]. The 
Jamie's Ministry of Food (JMOF) Australia was a community-based program that commenced in 2016 in Western Australia (WA), teaching basic cooking skills to help people prepare simple, fresh, healthy food quickly and cheaply. It therefore provided an excellent opportunity for a longitudinal study to examine the physiological benefits of food literacy and cooking programs and to evaluate their sustainability over time. This paper outlines the study protocol for the quantitative evaluation of the JMOF cooking program, it outlines participant recruitment, collection of data and proposed analysis of food literacy, dietary intake, physiological measurements and biomarkers of health.

JMOF was brought to communities across Australia by The Good Foundation (TGF). In Western Australia (WA) the program was delivered by TGF and supported by Edith Cowan University (ECU) as part of a three-year partnership. Through the use of a mobile kitchen, the program was delivered to communities across the state and involved a series of hands-on, basic, 7-week cooking classes led by a team of dedicated and nutritionally qualified Food Trainers who were supported by local volunteer ECU Student Interns. The cooking course was open to people aged 12 years and over, looking to learn the basics of cooking while having some fun in the kitchen with Jamie's hints, tips and shortcuts. To date there have been two previous evaluations of the program that were designed by [42-45] and included the online questionnaire that is used in this study. It featured questions specific to the JMOF program logic model [42] and was referred to in a subsequent peer review publication of JMOF evaluation by Herbert et al. [45]. The questionnaire contained the Rosenberg Self-Esteem Scale, which is a widely used and validated tool [46]. Standard evaluation information, consent, demographic details and participant contact details were also collected via the questionnaire. The underlying design of this study and the development of the questionnaire were based on the logic models described by Flego et al. [42] and were the property of the TGF. ECU collaborated with TGF to build on previous evaluation studies by including measurements of lifestyle and biomarkers of health to further explore the benefits of the program, however the basic design was limited to confines of the previous evaluations.

The aims of this study were to evaluate whether the JMOF program instigated a change to participants' skills, attitudes, knowledge, enjoyment and satisfaction of cooking and cooking confidence (self-efficacy). This aim was based on the premise that preventive nutrition, as delivered by a program such as the JMOF program, along with healthy connections to others, would improve quality of life and health outcomes with an associated minimisation of chronic disease risk factors that that would be sustained over time.
The primary evaluation explored cooking confidence (operationalised as personal beliefs of self-efficacy) and the ability to cook a meal from healthy ingredients. The secondary evaluation sought to determine whether improvements to self-efficacy influenced lifestyle and dietary intake and other underlying mechanisms that led to positive physiological and mental health and well-being, measured by biomarkers of chronic disease risk.

The study hypothesised that participation in a program to develop basic cooking skills and nutrition knowledge would improve cooking confidence (self-efficacy), that would impact lifestyle to improve dietary intake, thereby having a positive influence on physiological and mental health and their biomarkers.

\section{Methods \\ Study design}

The study has a pseudo-random, mixed-methods, prepost design involving an intervention group of participants who completed a cooking program that consisted of a 1.5-h session once a week for 7 weeks, in eight locations throughout WA. There was also a control group that comprised participants from these locations who were on the program wait-list (Fig. 1).

The study design was also premised upon the idea that participants would receive a continuity of support and education after attending the JMOF program. The qualitative component of the study incorporated formative research to determine what type of communication platform this could be delivered through. It involved interviews and focus groups with participants during the first round of the program who were asked about their program experiences, which provided insights that helped to explain the primary endpoints, as well as any impacts not captured by the quantitative measures. The outcome of which led to the development of a Facebook group that future participants could join, share their experiences and access educational links. Results from the qualitative components of this study have been reported but not published.

\section{Study participants}

Participants were selected from those enrolled in the cooking program who were community-dwelling adults, 18 years and above, who volunteered to participate in the evaluation of the cooking program; no other inclusion or exclusion criteria were applied. Ethics approval was provided by the ECU Human Research Ethics Committee (HREC) (ID 15362:Newton). All participants gave their written consent prior to commencement in the study. 
Study Diagram

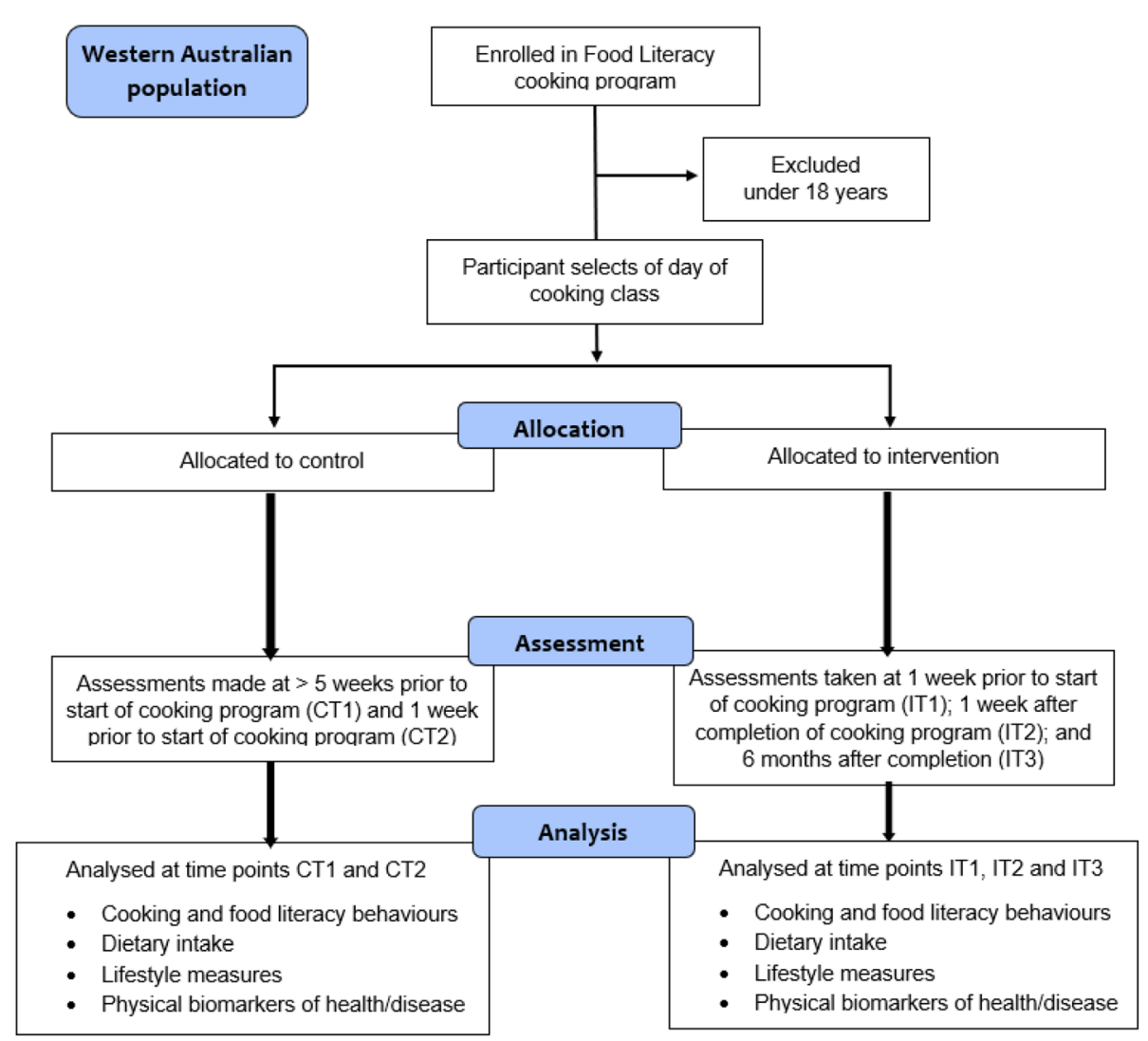

Fig. 1 Overview of the study design

\section{Recruitment strategy}

The study used a convenience sample of all participants 18 years and above, who registered for the JMOF program during the 3 years of its operation. A total of 19 courses were offered at various designated locations in WA over the 3 years and recruitment for these was conducted from $17 / 05 / 16$ to $1 / 11 / 18$. Each course was promoted via a portal on the JMOF website, firstly to the local community 3 months prior and secondly to the public 2 months prior to the designated start date. (https://www.jamiesministryoffood.com.au/courses).

Registration for each cooking program became available 6 weeks prior to its commencement. Metropolitan courses were held in the suburbs of Joondalup, Mt Lawley, Belmont, Mandurah, Sienna Wood and Baldivis and regional courses were held in Bunbury and Albany in South West WA. TGF managed the Australian website and the registration database for all courses. Reports of WA program registrants were sent to ECU Survey Research Centre (SRC) who contacted eligible adult participants to determine their interest to be involved in the study and the level at which they chose to participate.
For both control and intervention groups there were four levels of contribution, each building on the previous one by the inclusion of additional testing (Table 1). Those who opted into the study received a link to an online consent form and evaluation survey. Withdrawal was an option at any stage or level of participation.

\section{Assignment of groups}

Subsequent sampling for the control and intervention groups was purposive from the program registration database. Classes were offered on all days of the week except Sunday and the day chosen by the participant determined their allocation to either the control group or the intervention group. If a subject met the inclusion criteria of being 18 years and above and selected a class on either a Monday or a Thursday, they were eligible to become a control participant. Intervention participants were those who met the inclusion criteria of being 18 years and above and selected a class on any of the other days of the week. This sampling approach and allocation of registered people to the two groups was, therefore, pseudo-random and chosen to fit in with the design and 
Table 1 Participation level

\begin{tabular}{|c|c|c|c|c|c|}
\hline Level & $\begin{array}{l}\text { Online questionnaire } \\
\text { (includes, SF-12, SVS, } \\
\text { WEMWBS, IPAQ, GLTEQ, } \\
\text { and beverage intake) }\end{array}$ & FFQ (DQES v2) & $\begin{array}{l}\text { Anthropometry, blood pressure, } \\
\text { body composition, mobility, } \\
\text { agility and strength }\end{array}$ & $\begin{array}{l}\text { Blood and urine samples } \\
\text { (Lipids, glucose, hs-CRP, } \\
\text { IL-6, LPS, carotenoids, }\end{array}$ & $\begin{array}{l}\text { Stool samples } \\
\text { (SCFA, BA, } \\
\text { microbiome) }\end{array}$ \\
\hline 1 - Bronze & $\sqrt{ }$ & $x$ & $x$ & $x$ & $x$ \\
\hline 2 - Silver & $\sqrt{ }$ & $\sqrt{ }$ & $\sqrt{ }$ & $x$ & $x$ \\
\hline 3 - Gold & $\sqrt{ }$ & $\sqrt{ }$ & $\sqrt{ }$ & $\sqrt{ }$ & $x$ \\
\hline 4 - Platinum & $\sqrt{ }$ & $\sqrt{ }$ & $\sqrt{ }$ & $\sqrt{ }$ & $\sqrt{ }$ \\
\hline
\end{tabular}

(SF-12 The 12-item Short Form Survey, SVS Subjective Vitality Scale, WEMWBS Warwick-Edinburgh Mental Well-being Scale, IPAQ International Physical Activity Questionnaires, GLETQ Godin Leisure-Time Exercise Questionnaires, DQES FFQ Dietary Questionnaire for Epidemiological Studies Version 2 Food Frequency Questionnaire, hs-CRP High sensitivity C-Reactive Protein, IL-6 Interlukin-6, LPS Lipopolysaccharide, SCFA Short Chain Fatty Acids, BA Bile Acids)

philosophy of the program, which emphasises the importance of participants attending the program when and with whom they want.

\section{Intervention}

The intervention involved participation in one of the nineteen 7-week cooking programs that were delivered at six metro and two regional locations throughout WA from the fully equipped JMOF mobile kitchen. Each program involved a $1 \frac{1 / 2}{2}$ hour hands-on cooking session held once per week for 7 weeks. The weekly sessions were scaffolded in their recipe complexity to enable increased cooking confidence, skill development and food literacy knowledge. Classes were delivered by a JMOF-trained, qualified nutritionist who demonstrated the recipe of the day which participants then cooked for themselves in groups of four to a workstation. Each week there was a new "Jamie" recipe and over the course of the program participants learnt to cook dishes from every mealtime i.e. breakfast, lunch, dinner and side dishes. As well as learning cooking skills, the participants were taught knife handling as well as how to bring out the best flavours from fresh foods and how to create dishes without using pre-prepared ingredients. They were also taught how to plan a food budget to help reduce food waste and be more economic in the kitchen. Assessments for the Intervention group were undertaken just prior to their program start date, immediately after their program was completed and 6 months after completion (Fig. 2).

\section{Control}

The control group's assessments were undertaken during the six-week period leading up to their participation in the cooking program, thereby representing those who had not undergone the intervention (Fig. 2).

\section{Primary and secondary outcomes and endpoints}

The primary outcome of cooking confidence was evaluated via the theoretical concept of personal beliefs of self-efficacy measured across five areas:
1. Confidence about being able to cook from basic ingredients;

2. Confidence about following a simple recipe;

3. Confidence about preparing and cooking new foods and recipes;

4. Confidence that what is cooked will "turn out" well; and

5. Confidence about tasting foods not eaten before.

This was performed using the same method as described by Flego et al. [42] by measuring cooking confidence as a proxy for self-efficacy. The questions used were based on works by Short (2003) and Barton (2011) and presented on a 5-point Likert confidence scale ranging from 'not at all' to extremely' confident. This was explored in terms of change over time and between different study groups.

The secondary endpoints were measures of dietary intake, physical activity and mobility levels, body composition, anthropometry, blood, urine and faecal biomarkers of systemic, physical and mental health and are further explained in the sections below. These were also explored in terms of change over time and between different study groups.

\section{Sample size}

The sample size calculation in this study was based on the findings of the first published quantitative evaluation of Jamie's Ministry of Food (JMOF) program into its impact on participants' cooking confidence [42]. Flego et al. reported an increase of $>0.53$ on a 5-point Likert scale across all confidence items, which corresponded to a large Cohen's effect $(\mathrm{d}>0.8)$, whilst no significant changes were observed in the Control group (all $p>0.13$ ). Using G*Power, the required sample for a mixed-model analysis of variance test (ANOVA) design (2 groups, each with 2 time points) to detect (conservatively) a smallmedium, within-between, interaction effect (Cohen's $f=$ 0.15 ), at the $5 \%$ level of significance and $80 \%$ power was 90, or 45 per group. However, Flego et al. [42] noted that the participant retention rate in the JMOF program at T2 was 55\% (694 at $\mathrm{T} 1$ and 383 at T2). To account for this 


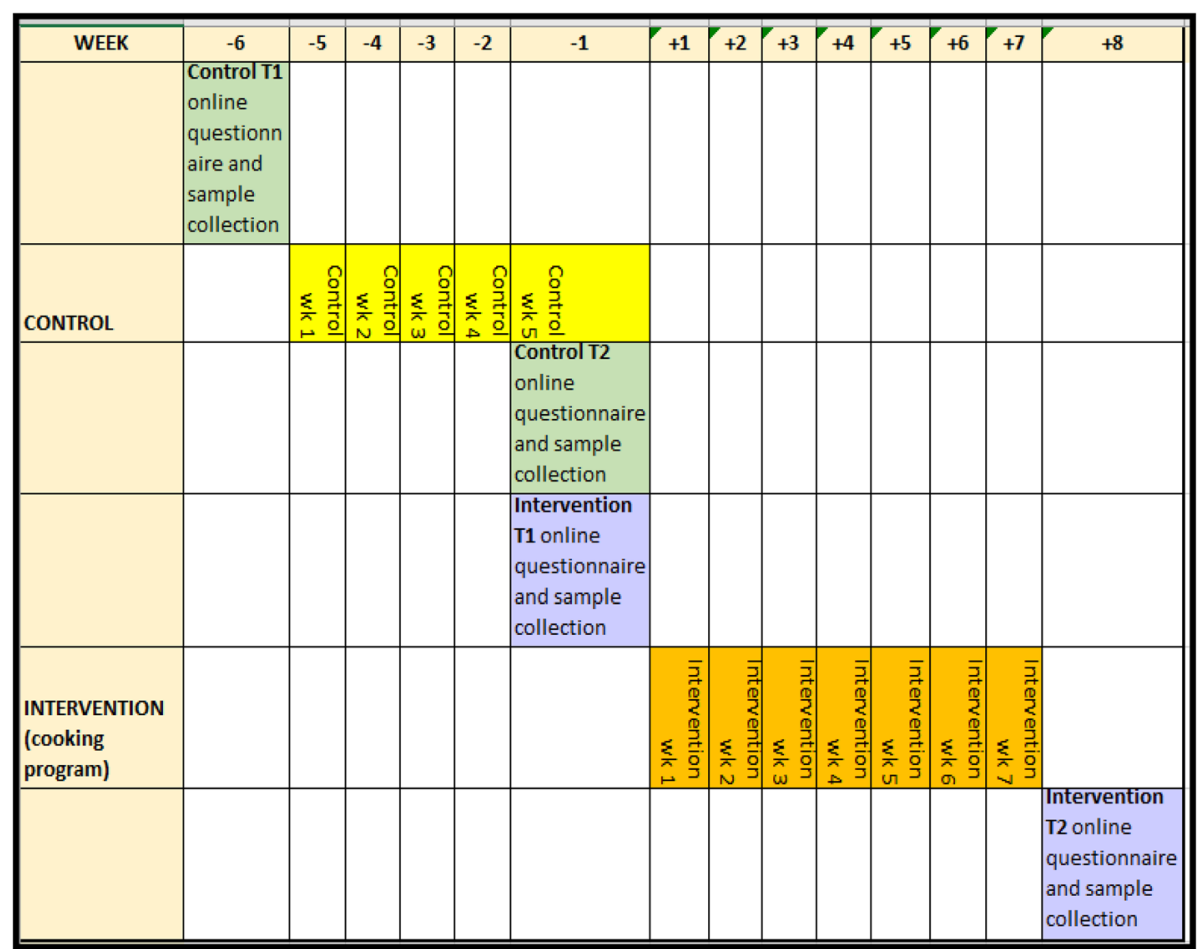

Fig. 2 Study timeline

and to ensure sufficient participation numbers at the end of T2, the aim was to recruit a minimum of 164 participants with 87 in each time-point (i.e. T1 and T2) of the intervention arm. No power calculation to account for $\mathrm{T} 3$ samples size was conducted as the investigation was not aiming to detect a change, rather, whether the effect was maintained at $\mathrm{T} 3$.

\section{Data collection schedule and measures}

At all levels of contribution, intervention participants were surveyed at three time points: before the program start (IT1), on program completion (IT2), and 6 months after program completion (IT3). Control participants were surveyed at two time points: 6 weeks prior to program commencement (CT1) and on entry to the program (CT2) (Fig. 1). Computer programmed 'rules' automated this process. The ECU SRC ensured consent prior to participation in the study and distributed the online survey assessment using Voxco CATI@ Software [47]. The ECU SRC complies with the AMSRS Code of Professional Behaviour and since 2008 SRC has been an ISO20252 standards accredited organisation. All projects undertaken by ECU SRC are conducted in full compliance with the Interviewer Quality Control Australia (IQCA) as recommended by the Australian Market Research Industry and NHMRC/AVCC guidelines on research practices. Postal versions of all documentation were sent to persons who did not have a working email address or access to a computer. The ECU SRC sent participants their online questionnaire 1 week prior to T1, T2 and T3 (for intervention) and if necessary, they received a second reminder email during the week of $\mathrm{T} 1, \mathrm{~T} 2$ or $\mathrm{T} 3$ as well as a clinic appointment reminder where applicable.

Four levels of contribution were offered to all WA adults registered on the program database (Table 1). The minimum level of contribution, completed by participants at all levels at each time point, consisted of the basic online survey evaluation used in Victoria and NSW [42-45] with the incorporation of additional assessments. These included, quality of life measured using the SF- $12^{\circ}$ Health Survey [48], wellness using the Subjective Vitality Scale $[49,50]$ and the Warwick-Edinburgh Mental Well-being Scale (WEMWBS) [51], and physical activity determined using the International Physical Activity Questionnaire (IPAQ) self-administered short-form [52] and the Godin LeisureTime Exercise Questionnaire [53] (Additional file 1). Those who chose to contribute at level 2 completed the above questionnaire and also attended a clinic appointment at each time point, where they underwent dietary, anthropometric, body composition, blood pressure, timed "Up \& Go" [54] and hand grip strength [55] assessments.

\section{Dietary assessment}

Participants completed a hard copy version of the Dietary Questionnaire for Epidemiological Studies Version 2, 
food frequency questionnaire (DQES v2, FFQ) developed by Cancer Council Victoria [56] at each time point to assess dietary intake and alcoholic beverage consumption. This questionnaire includes 100 different food items and was designed to assess the usual frequency of dietary intakes over a 12-month period, with 10 frequency response options ranging from 'never' to ' 3 or more times per day'. Portion size was calculated using photographs of scaled portions of different food types. Energy $(\mathrm{kJ} / \mathrm{d})$ and 31 different intakes of nutrient were calculated by using the NUTTAB95 food composition database [57]. To explore changes after 7 weeks and 6 months for the intervention group and after 5 weeks for the control group the FFQ was re-administered with emphasis on dietary intake in the period being explored. The use of this questionnaire has been validated by Hebden et al. [58] and Petersen et al. [59], however to further explore dietary intake of fruit and vegetables, plasma carotenoids will be measured from participants' blood samples [5862]. The use of fresh and healthy ingredients reported by intakes of fruit and vegetables were used to estimate intakes of insoluble fibre, soluble fibre, resistant starch and polyphenols. Dietary fibre, resistant starch and polyphenols are of particular interest due to their interaction with the gut microbiota and their resulting influence on health [62-66].

Similarly, participants completed a short beverage questionnaire that determined the number of cups/ glasses of a range of beverages regularly consumed (average over 4 weeks and daily amount), that were used to estimate polyphenol intake from beverages.

\section{Anthropometry}

Anthropometric measurements included height (m), weight $(\mathrm{kg})$, body mass index (BMI) $\left(\mathrm{kg} / \mathrm{m}^{2}\right)$ and waist circumference $(\mathrm{cm})$. Standing height and body mass were measured to the nearest $0.1 \mathrm{~cm}$ and $0.1 \mathrm{~kg}$ respectively with a SECA 763 digital column scale (SECA Ltd., USA). Waist circumference was measured at the narrowest part of the waist by a Lufkin steel tape measure to the nearest $0.1 \mathrm{~cm}$. All measurements were conducted according to standardised ISAK techniques [67].

\section{Body composition}

Body Composition was measured using the BOD POD (air displacement plethysmography) (COSMED AsiaPacific Pty Ltd., Artarmon NSW) The BOD POD uses whole-body densitometry to determine body composition in terms of fat and fat-free mass. All participants who opted for level 2 and above, underwent two tests at each visit lasting approximately $50 \mathrm{~s}$ each. The same BOD POD machine was used throughout the study and was calibrated and maintained as per the manufacturer's recommendations. In some cases, additional measurements of regional and whole-body lean mass and fat mass were derived from a whole-body scan with Dual-Energy X-ray Absorptiometry (DXA) (Hologic 4500A Discovery, Hologic Corp., Waltham, MA, USA). The level of radiation exposure from the DXA scans was negligible (10-30 microSieverts $[\mu \mathrm{Sv}]$ in comparison to a flight from Perth to London, approximately $100 \mu \mathrm{Sv}$ ). The number of scans conducted in this study were well within the guidelines provided by the manufacturer of the DXA. The scans were conducted by a qualified operator using the same machine each time, however as only a limited number of participants underwent these tests, the results are for reference only.

Due to the inability to relocate the BOD POD machine, participants who attended regional clinics had their body composition, fat mass and fat-free mass measured to the nearest $0.1 \%$ using a transportable Impedimed Imp DF50 (Impedimed Ltd., QLD) tetrapolar bioelectrical impedance analyser (BIA). For this procedure participants were asked to lie in a supine position and four electrodes were placed on their hands and feet. A weak electrical current was passed through their body (which they could not feel) and readings from this were used to calculate fat mass, fat-free mass and body water. Reliability of body composition can be influenced by level of hydration. Participants were asked to attend the clinics well hydrated, to refrain from water/fluid ingestion for $1 \mathrm{~h}$ prior to the scan and void their bladder.

\section{Blood pressure}

An Omron IA1B Automated Blood Pressure Device (Omron Healthcare Ltd., Japan) was used to measure participants' blood pressure. Readings were taken in triplicate with the participant in a calm and relaxed state, in a seated position, on their arm at heart level, at 1-min intervals. All three readings were recorded with the lowest to be used in analysis.

\section{Timed "Up \& Go" test}

Dynamic balance and agility (i.e. functional mobility) were measured using the timed "Up \& Go" test [54]. The test quantifies the time it takes to stand up from a seated position in a 'standard' chair and walk around a mark placed $3 \mathrm{~m}$ from the chair, and then sit back down on the chair. Timing was started as the participant stood up and was stopped once they were re- seated clearly on the chair in an upright position. After familiarisation, one properly executed trial was allowed with time recorded and reported to the nearest $0.01 \mathrm{~s}$.

\section{Grip strength}

Hand grip strength $(\mathrm{kg})$ was obtained using a Jamar Analogue Hand Dynamometer on the dominant hand [55]. The participant stood with arms by their side and 
elbow bent at 90 degrees and when instructed to do so, squeezed the dynamometer with as much force as possible. Three trials lasting $3 \mathrm{~s}$ each were completed with $15 \mathrm{~s}$ rest between them and the maximum value was used for analysis.

\section{Measures of biochemistry and urinalysis}

Cumulating on level 2 assessments, those who selected the third level of contribution also provided blood and urine samples for biochemical analysis at each time point. Blood and urine samples were collected by trained phlebotomists in the morning after an overnight fast. The venous blood samples were centrifuged and processed within $30 \mathrm{~min}$ after collection to separate plasma and serum. These were then aliquoted into 1.5 or $2 \mathrm{~mL}$ vials and stored at -80 degrees until analysis. Biochemical variables related to cardiovascular, liver, cognitive and musculoskeletal disease such as fasting blood lipids, glucose and high sensitivity $\mathrm{C}$-reactive protein will be measured by routine laboratory methods. Carotenoid concentration ( $\alpha$-carotene, $\beta$-carotene, lycopene, $\beta$ cryptoxanthin, lutein) will be analysed as objective indicators of fruit and vegetable intake [68]. Participants gave their permission for future analyses that include the following but are dependent on available funding; advanced glycation end products (IgG $N$-glycans) measured to assess early signs of health risks such as hypertension, inflammatory diseases and cardiovascular diseases [69]; serotonin and lipopolysaccharide (LPS) as biomarkers of mental and gut health [70, 71].

\section{Human faecal sample collection, processing and analysis}

The fourth and maximum level of contribution incorporated the additional provision of a 24-h stool sample at each time point. Instructions and equipment were provided for the collection of stools from all bowel motions over a $24 \mathrm{~h}$ period, if more than one stool sample was collected, they were homogenised as individual samples and then pooled and homogenised again. This was done to provide a more accurate, homogenous measure of the gut microbiota and gut metabolites. Participants were given a portable cooler bag and frozen icepacks to store the stool samples over the $24 \mathrm{~h}$ collection period and to return their sample(s) to the clinic. Once received the frozen stool samples were weighed and stored at -80 degrees. Stool samples were thawed in a fridge ( $\max$ $4{ }^{\circ} \mathrm{C}$ ) and homogenised before being aliquoted for various analyses. Stool aliquots for short chain fatty acids (SCFA), bile acids and microbial analysis remain at $80^{\circ} \mathrm{C}$ until their analysis.

Microbial analyses will be performed at the WA Human Microbiome Collaboration Centre, Perth WA. DNA will be extracted using the QIAamp PowerFecal DNA kit (Qiagen) using Qiacube extraction platform.
Bacterial signatures are generated using the Illumina MiSeq platform using uniquely barcoded $16 \mathrm{~S}$ rRNA gene primers (515-806(V4)), following polymerase chain reaction (PCR) inhibition assessment of each DNA extract. PCR-free ligation protocol is thereafter deployed for library building. Samples will be sequenced to a depth of minimum 50,000 reads, which is sufficient to identify microbes to a genus/species level. Quality control and mock community samples are included in the analysis from sample collection to sequence analysis. Sequence read quality is initially assessed with FastQC before demultiplexing and pre-processing by GHAPv2, an inhouse tool. Cutadapt [72] is used for removal of all nonbiological sequences. DADA2 [73] is then used for quality filtering, error correction and amplicon sequence variants (ASVs) picking. A trained naïve bayes classifier then assigns the ASV's to genus/species against a curated database of microbial reference sequences such as the RDP [74] or Genome Taxonomy Database [75].

Measures of dietary fibre and protein fermentation in the stool samples will be determined from short chain fatty acids (SCFA) using gas chromatography mass spectrometry at ECU. SCFA in faecal material will be extracted into an acidified aqueous methanol solution and the SCFA separated by gas chromatography using a fatty acid column (Zebron ZB-FFAP $30 \mathrm{~m} \times 0.53 \mathrm{~mm} \times 1 \mu \mathrm{m}$ supplied by Phenomenex). The SCFA will be detected using mass spectrometry and their concentrations determined by the method of internal standards. Isotopically internal standards will be spiked into the extraction solution.

As high fat diets have been associated with poorer health outcomes, in addition to blood lipids, high performance liquid chromatography in tandem with mass spectrometry will be used to analyse faecal bile acids. Beyond their role of facilitating fat digestion, recent research discoveries have emphasised the importance of bile acid metabolism as a regulator of human physiology and central to overall health [76, 77] and disruptions to BA processing are associated with the onset of chronic disease. The target bile acids will be extracted from the faecal material by solid phase extraction and then injected into a C18 column. The separated bile acids will be identified and quantified by mass spectrometry using a method developed by ECU and adapted from $[78,79]$. The method of internal standards will be adopted to correct for matrix effects.

The SPIRIT figure for study protocols is presented in Fig. 3 and the 33 item SPIRIT checklist in Additional file 2 (separate upload) [80, 81].

\section{Data management}

Data management (collection, recording, and storage) complies with the rules of ECU Human Research Ethics 


\begin{tabular}{|c|c|c|c|c|c|c|c|c|}
\hline & Enrolment & Allocation & & & & $\begin{array}{l}\text { Cooking } \\
\text { Program }\end{array}$ & & \\
\hline TIMEPOINT & & 0 & $\begin{array}{l}C t_{1} \\
-6 w k\end{array}$ & $\begin{array}{l}\mathrm{Ct}_{2} \\
- \\
1 w k\end{array}$ & $\begin{array}{l}I_{1} \\
- \\
1 w k\end{array}$ & $1-7 w k$ & $\begin{array}{l}I t_{2} \\
8 w k\end{array}$ & $\begin{array}{l}\text { It } 3 \\
+6 m o\end{array}$ \\
\hline \multirow{3}{*}{$\begin{array}{l}\text { Eligibility screen } \\
\text { Informed consent } \\
\text { Allocation }\end{array}$} & $\mathrm{XX}$ & & & & & & & \\
\hline & $\mathrm{XX}$ & & & & & & & \\
\hline & & $X X$ & & & & & & \\
\hline INTERVENTION: & $x$ & $\mathrm{X}$ & & & $\mathrm{X}$ & $\mathrm{x}$ & $X$ & $\mathrm{X}$ \\
\hline CONTROL: & $\mathrm{X}$ & $\mathrm{X}$ & & & & $\mathrm{X}$ & & \\
\hline \multirow{5}{*}{$\begin{array}{l}\text { ASSESSMENTS: } \\
\text { Baseline demographic } \\
\text { characteristics and } \\
\text { online questionnaire (all } \\
\text { levels) } \\
\text { Dietary, anthropometric, } \\
\text { body composition, blood } \\
\text { pressure, mobility and } \\
\text { strength assessments } \\
\text { (Levels } 2-4 \text { ) } \\
\text { Biochemistry and } \\
\text { urinalysis biomarkers } \\
\text { (levels } 3 \text { \& 4) } \\
\text { Faecal biomarker } \\
\text { analysis (level 4) }\end{array}$} & & & & & & & & \\
\hline & & & $X$ & $X$ & $\mathrm{X}$ & & $\mathrm{x}$ & $x$ \\
\hline & & & $\mathrm{x}$ & $x$ & $\mathrm{x}$ & & $x$ & $\mathrm{X}$ \\
\hline & & & $x$ & $X$ & $x$ & & $x$ & $x$ \\
\hline & & & X & $X$ & X & & X & X \\
\hline
\end{tabular}

Fig. 3 SPIRIT figure for study protocols

Committee (HREC) and is reported on annually (ID 15362:Newton). All data has been de-identified and stored according to HREC policy in access restricted, secure repositories.

\section{Statistical methods}

The computer software package IBM SPSS Statistics for Windows version 25.0 (Armonk, NY: IBM Corp) will be used to access the data set and perform the statistical analyses. For the basic online evaluation questionnaire, general descriptive analyses including frequency data with appropriate plots and cross-tabulations will be used to explore and summarise outcome variables. Continuous demographic and baseline characteristics will be summarised using standard statistics (mean and standard deviation) and non-parametric statistics (medians and inter-quartile ranges) where appropriate. Independent $t$-test for normally distributed data, and MannWhitney $U$ test for non-normal data, will be used to assess differences between the groups. Frequencies and percentages will be reported for categorical variables and Chi-square test will be used to test for differences in proportion between the groups. The magnitude of change both within and between the intervention and control groups will be assessed using mixed-model ANOVA. Furthermore, changes within the intervention group will be assessed across three time points IT1, IT2 and IT3 using repeated-measures ANOVA. To account for multiple outcomes, the Benjamini-Hochberg correction (i.e. false discovery rate) will be applied to the raw $p$-values. Participants with missing value for the variables of interest (either outcome or predictor) will be removed from the repeated-measures ANOVA models. Specific demographic factors of interest (including but not limited to, gender, age, education, BMI and employment) will be adjusted for in all models. Statistical significance will be indicated at the 10, 5 and $1 \%$ level. Cohen's effect sizes will be provided as a measure of practical/clinical significance and to assist in the interpretation of said 'statistically significant' results.

To interpret gut microbiota diversity and to analyse the microbial composition of the gut microbiota, multivariate analysis using a combination of $\mathrm{R}$ and PRIMER7 and Permanova+ (PRIMER-E, Plymouth) [82] will be conducted. Principal Coordinates Analysis (PCoA) will be deployed to visualise findings. Distance-based linear models (DISTLM) and distance-based redundancy analysis (dbRDA) will be used to integrate microbiome findings with other relevant data (i.e. dietary intake) that help explain the relationship between the microbiome findings and other outcomes.

\section{Discussion}

It is well reported that an increased emphasis on families and individuals to change their dietary patterns and lifestyle can have significant impact on weight reduction and overall health [29, 83-87]. Family dinners are have been found to be a measurable signature of these social exchanges in the home that support socioemotional development and mental health [88]. A 2017 study reported an association between increased frequency of family dinners and lower rates of obesity that was pronounced for those whose meals were home-cooked [89]. Indeed, the consensus statement of the International 
Society for Nutritional Psychiatry Research (ISNPR) stresses the importance of diet and nutrition as a critical determinant of mental and physical health [90]. The collaboration of academics advocates for future public health interventions to stimulate a significant change in eating habits back to more traditional wholefood diets.

Yet, research in WA suggests that the demands on families [91-93], together with fatigue [93], lack of time, family/social support and overall motivation [91, 93, 94], impede prioritisation of a healthy diet and lifestyle [95].

The JMOF mobile kitchen has been delivering sevenweek cooking programs to the community teaching basic cooking skills aimed at improving participants' knowledge and ability to purchase, prepare and cook healthier meals at home. This study protocol describes the recruitment of willing participants and the assessment methods involved in an evaluation of the program's effect on their cooking confidence, dietary intakes, physical and mental health and wellbeing. The study extends on previous, similar studies in that it includes collection and analysis of biomarkers of both metabolic and gut health. The study will provide valuable insight into the effectiveness of food literacy cooking programs and how improvements to cooking confidence and skills can contribute to healthier outcomes.

Strengths of the study include the extensive range of data collected that comprise; self-reported cooking confidence and self-efficacy and measures of physical and mental health and well-being. In addition, data has been collected on self-reported dietary intake; measures of health status from anthropometry, body composition, blood pressure, agility and grip strength, blood, urine and faecal biomarkers. The study has also collected longitudinal data to explore sustainability. Findings will support future public health cooking program initiatives aimed at improving food literacy, self-efficacy and physical and mental health. It is also novel in that it will help to identify mechanistic associations between dietary intake and interactions between human gut microbiota, their metabolites and their interplay with host metabolism in both physiological and mental health.

There is burgeoning evidence reporting the beneficial associations between the human gut-microbiome and the key microbes and their metabolites that are associated with health $[18,19]$. Diets higher in protein and fats and lacking in fermentable substrates for the production of beneficial SCFA have increasingly been linked to poorer physical and mental health outcomes [65, 96-98] and the presence of microbial profiles that are more often associated with disease [76]. The inclusion of faecal bile acid analysis will enable investigation of their role in human metabolism beyond their core function of assisting with dietary fat digestion and absorption. Faecal bile acid composition is of great interest when studying the role of diet and gut microbiota in health and disease, due to the bi-directional interplay between gut bacteria and bile acid and cholesterol metabolism that leads to implications on host physiology [76, 99].

The study is limited due to the confines of the study design that prevented true randomisation. According to the Stages of Change theory [100], as the study participants were selected from the wait-list and had already committed to the program, they would have transitioned from the pre-contemplation to the change/action stage. Therefore the act of registering for the program could potentially instigate behaviour change, which may result in positive change within both groups [101]. As a consequence, the control group may not truly reflect the general population. The standard evaluation used the tool created for previous Australian cooking programs that were run in Victoria and NSW [42-44], however this study extended data collection to include more detailed assessments of physical and mental health as described in this protocol. The DQES vs 2 FFQ is a reflection of intake over 12 months, however it has been validated to detect change over a shorter timeframe in similar studies $[58,59]$.

The study's strengths include the extensive data collected that will provide valuable insight to the effects that a basic cooking program incur within a healthy population of Australian adults. Findings will inform on the complex interaction between self-efficacy, dietary intake, gut-microbiota and biomarkers of physiological health and well-being, that may support the employment of basic cooking programs as a tool for future health promotion interventions.

\section{Supplementary information}

Supplementary information accompanies this paper at https://doi.org/10. 1186/s12889-020-09124-3.

Additional file 1. Additional information [48-53, 102]

Additional file 2. SPIRIT checklist

\section{Abbreviations}

ANZCTR: Australian and New Zealand Clinical Trials Registry; ABS: Australian Bureau of Statistics; DF: dietary fibre; JMOF: Jamie's Ministry of Food; WA: Western Australia; TGF: The Good Foundation; ECU: Edith Cowan University; HREC: Human Research Ethics Committee; SRC: Survey Research Centre; IQCA: Interviewer Quality Control Australia; ANOVA: analysis of variance test; WEMWBS: Warwick Edinburgh Mental Well Being Scale; IPAQ: International Physical Activity Questionnaire; DQES vs 2 FFQ: Dietary Questionnaire for Epidemiological Studies Version 2, food frequency questionnaire; BMI: body mass index; DXA: Dual-Energy X-ray Absorptiometry; BIA: bioelectrical impedance analyser; LPS: lipopolysaccharide;

PCR: polymerase chain reaction; ASVs: amplicon sequence variants; SCFA: short chain fatty acids; PCOA: Principal Coordinates Analysis; DISTLM: distance-based linear models; dbRDA: distance-based redundancy analysis; $\mathrm{NaOH}$ : Sodium Hydroxide

\section{Acknowledgements}

The authors of this report acknowledge all the staff of The Good Foundation (including staff of the Mobile Kitchen WA) for assisting with the implementation of the evaluation, and to all participants who kindly offered their time to participate and complete the evaluation questionnaires. 


\section{Authors' contributions}

$A D, C C, R N, M F B, R S$ and $L C$ contributed to the conception and design of the study. JR, AD, CC and CW were responsible for implementation. JL provided statistical expertise, JR is a grant holder and JR, CC, JRL, JL, MCB, LC and $A D$ contributed to refinement of the protocol and the writing and editing of the manuscript. All authors read and approved the final manuscript.

\section{Funding}

This research received no external funding from any agency in the public, commercial, or not-for-profit sectors. The study was self-supported by ECU, as the Major Partner - Western Australia, by provided funding to The Good Foundation for the provision of a Jamie's Ministry of Food Australia Mobile Kitchen at ECU campuses and other agreed Western Australian locations across a 3-year period. ECU also funded all utility costs associated with the program when held at ECU campuses, and the research study in its entirety. Beyond that, the salary of JRL is supported by and National Health and Medical Research Council of Australia Career Development Fellowship (ID: 1107474).

\section{Availability of data and materials}

The datasets generated and/or analysed during the current study are not publicly available due to there being a consulting services deed agreement between ECU and The Good Foundation that prohibits the disclosure of data for this study. Data are however available from the authors upon reasonable request and with permission of The Good Foundation.

\section{Ethics approval and consent to participate}

This study has been approved by the Edith Cowan University Human Research Ethics Committee (HREC) (ID 15362:Newton). All participants were provided with a either a digital or hard copy written consent form which they signed prior to commencement in the study.

\section{Consent for publication}

Not applicable.

\section{Competing interests}

The authors declare they have no competing interests.

\section{Author details}

${ }^{1}$ School of Medical and Health Sciences, Edith Cowan University, 270 Joondalup Drive, Joondalup, Perth, WA 6027, Australia. ${ }^{2}$ WA Human Microbiome Collaboration Centre, School of Molecular \& Life Sciences, Curtin University, Perth, WA, Australia. ${ }^{3}$ Medical School, University of Western Australia, Perth, Australia. ${ }^{4}$ School of Public Health, University of Sydney, Sydney, Australia. ${ }^{5}$ School of Science, Edith Cowan University, Perth, WA, Australia. ${ }^{6}$ School of Education, Edith Cowan University, Perth, WA, Australia. ${ }^{7}$ Centre for Integrated Metabolomics and Computational Biology, Edith Cowan University, Perth, WA, Australia. ${ }^{8}$ Exercise Medicine Research Institute, Edith Cowan University, Perth, WA, Australia. ${ }^{9}$ School of Human Movement and Nutrition Sciences, University of Queensland, Brisbane, QLD, Australia.

Received: 6 March 2020 Accepted: 16 June 2020

Published online: 30 June 2020

\section{References}

1. Von Ruesten A, Feller S, Bergmann MM, Boeing H, Sahlgrenska A. Institute of Medicine DoPH, et al. diet and risk of chronic diseases: results from the first 8 years of follow-up in the EPIC-Potsdam study. Eur J Clin Nutr. 2013; 67(4):412-9.

2. Woodside JV, Young IS, McKinley MC. Fruit and vegetable intake and risk of cardiovascular disease. Proc Nutr Soc. 2013;72(4):399-406.

3. Asif M. The prevention and control the type-2 diabetes by changing lifestyle and dietary pattern. J Educ Health Promotion. 2014;3:1.

4. Arem H, Reedy J, Sampson J, Jiao L, Hollenbeck AR, Risch H, et al. The healthy eating index 2005 and risk for pancreatic cancer in the NIH-AARP study. J Natl Cancer Inst. 2013;105(17):1298-305.

5. MacFarlane A, Cleland V, Crawford D, Campbell K, Timperio A. Longitudinal examination of the family food environment and weight status among children. Int J Pediatr Obes. 2009;4(4):343-52.
6. Guthrie JF, Lin B-H, Frazao E. Role of food prepared away from home in the American diet, 1977-78 versus 1994-96: changes and consequences. J Nutrition Educ Behavior. 2002;34(3):140.

7. Jaworowska A, Blackham T, Davies IG, Stevenson L. Nutritional challenges and health implications of takeaway and fast food. Nutr Rev. 2013;71(5): 310-8.

8. Australian Institute of Health and Welfare. Australia's Health 2016: in brief. Canberra: AlHW; 2016. Report No.: Cat.no. AUS 201.

9. Van der Horst K, Brunner T, Siegrist M. Ready-meal consumption: associations with weight status and cooking skills. Public Health Nutr. 2011; 14(2):239-45.

10. ABS. National health survey, first results, 2017-18. Canberra: ABS; 2018. Report No.: 4364.0.55.001.

11. Australian Institute of Health and Welfare. A picture of overweight and obesity in Australia 2017. Canberra: AlHW; 2017. Report No.: PHE 216.

12. Oddy WH, Allen KL, Trapp GSA, Ambrosini GL, Black L, Huang RC, et al. Dietary patterns, body mass index and inflammation: pathways to depression and mental health problems in adolescents. Brain Behav Immun. 2018;69:428-39.

13. Koponen H, Jokelainen J, Keinanen-Kiukaanniemi S, Vanhala M. Depressive symptoms and 10-year risk for cardiovascular morbidity and mortality. World J Biol Psychiatry. 2010;11(6):834-9.

14. Lassale C, Batty GD, Baghdadli A, Jacka F, Sanchez-Villegas A, Kivimaki M, et al. Healthy dietary indices and risk of depressive outcomes: a systematic review and meta-analysis of observational studies. Mol Psychiatry. 2019;24:965-86.

15. Li Y, Lv MR, Wei YJ, Sun L, Zhang JX, Zhang HG, et al. Dietary patterns and depression risk: a meta-analysis. Psychiatry Res. 2017;253:373-82.

16. Osborn DJ, Levy G, Nazareth I, Petersen I, Islam A, King MB. Relative risk of cardiovascular and cancer mortality in people with severe mental illness from the united kingdom\&\#39;s general practice research database. Arch Gen Psychiatry. 2007;64(2):242-9.

17. Sonnenburg ED, Sonnenburg JL. Starving our microbial self: the deleterious consequences of a diet deficient in microbiota-accessible carbohydrates. Cell Metab. 2014;20(5):779-86.

18. Boulange CL, Neves AL, Chilloux J, Nicholson JK, Dumas ME. Impact of the gut microbiota on inflammation, obesity, and metabolic disease. Genome Med. 2016;8(1):42.

19. Newsholme P, Homem de Bittencourt PI Jr. Gut associated bacteria are critical to metabolism, inflammation and health. Curr Opin Clin Nutr Metab Care. 2016;19(4):245-9.

20. McLeod E, Campbell K, Hesketh K. Nutrition knowledge: a mediator between socioeconomic position and diet quality in Australian firtst-time mothers. J Am Diet Assoc. 2011;111(5):696-704.

21. Butcher L, Ryan M, O'Sullivan T, Lo J, Devine A. What drives food insecurity in Western Australia? How the perceptions of people at risk differ to those of stakeholders. Nutrients. 2018;10(8):1059.

22. Cleland V, Hume C, Crawford D, Timperio A, Hesketh K, Baur L, et al. Urbanrural comparison of weight status among women and children living in socioeconomically disadvantaged neighbourhoods. Med J Aust. 2010;3(192): $137-40$.

23. Foley W, Spurr S, Lenoy L, De Jong M, Fichera R. Cooking skills are important competencies for promoting healthy eating in an urban indigenous health service. Nutrition Dietetics. 2011;68(4):291-6.

24. Begley A, Paynter E, Butcher LM, Dhaliwal SS. Examining the Association between Food Literacy and Food Insecurity. Nutrients. 2019;11(2):445.

25. Butcher LM, Ryan MM, O'Sullivan TA, Lo J, Devine A. Food-insecure Household's self-reported perceptions of food labels. Product Attributes Consumption Behaviours. 2019;11(4):828.

26. Cullerton K, Donnet T, Lee A, Gallegos D. Using political science to progress public health nutrition: a systematic review. Public Health Nutr. 2015;9(11): 2070-78.

27. Garcia AL, Vargas E, Lam PS, Shennan DB, Smith F, Parrett A. Evaluation of a cooking skills programme in parents of young children: a longitudinal study. Public Health Nutr. 2014;17(5):1013-21.

28. Keller HH, Gibbs A, Wong S, Vanderkooy P, Hedley MJJNE. Men can cook! Development, implementation, and evaluation of a senior Men's Cooking Group; 2004. p. 24.

29. Mills S, Brown H, Wrieden W, White M, Adams J. Frequency of eating home cooked meals and potential benefits for diet and health: cross-sectional analysis of a population-based cohort study. Int J Behav Nutr Phys Act. 2017;14(1):109. 
30. Reicks $M$, Kocher $M$, Reeder J. Impact of cooking and home food preparation interventions among adults: a systematic review (2011-2016). J Nutr Educ Behav. 2018;50(2):148-72 e1.

31. Block K, Johnson B. Evaluation of the Stephanie Alexander Kitchen Garden Program. Final Report to: The Stephanie Alexander Kitchen Garden Foundation.: The Stephanie Alexander Kitchen Garden Foundation.; 2009.

32. Fulkerson JA, Rydell S, Kubik MY, Lytle L, Boutelle K, Story M, et al. Healthy Home Offerings via the Mealtime Environment (HOME): feasibility, acceptability, and outcomes of a pilot study; 2010. p. 18.

33. Bodenlos JS, Wormuth BM. Watching a food-related television show and caloric intake. A laboratory study. Appetite. 2013;61(1):8-12.

34. Engler-Stringer $\mathrm{R}$, Berenbaum SJCJCMH. Exploring social support through collective kitchen participation in three Canadian cities 2007;26.

35. Rees R, Hinds K, Dickson K, O'Mara-Eves A, Thomas J. Communities that cook: a systematic review of the effectiveness and appropriateness of interventions to introduce adults to home cooking. EPPI-Centre report 2004 London: EPPI-Centre, Social Science, Research Unit, Institute of Education, University of London; 2012

36. lacovou M, Pattieson DC, Truby H, Palermo C. Social health and nutrition impacts of community kitchens: a systematic review. Public Health Nutr. 2013;16(3):535-43.

37. Garcia AL, Reardon R, McDonald M, Vargas-Garcia EJ. Community interventions to improve cooking skills and their effects on confidence and eating behaviour. Curr Nutr Rep. 2016;5(4):315-22.

38. Fulkerson JA, Farbakhsh K, Lytle L, Hearst MO, Dengel DR, Pasch KE, et al. Away-from-home family dinner sources and associations with weight status, body composition, and related biomarkers of chronic disease among adolescents and their parents. J Am Diet Assoc. 2011;111(12):1892-7.

39. Fulkerson JA, Kubik MY, Rydell S, Boutelle KN, Garwick A, Story M, et al. Focus groups with working parents of school-aged children: what's needed to improve family meals? J Nutr Educ Behav. 2011;43(3):189-93.

40. Harman V, Cappellini B. Mothers on display: lunchboxes, social class and moral accountability. Sociology. 2015;49(4):764-81.

41. Connors M, Bisogni CA, Sobal J, Devine C. Managing values in personal food systems. Appetite. 2001;36(3):189-200.

42. Flego A, Herbert J, Gibbs L, Swinburn B, Keating C, Waters E, et al. Methods for the evaluation of the Jamie Oliver Ministry of Food Program, Australia. BMC Public Health. 2013;13(411). https://doi.org/10.1186/1471-2458-13-411.

43. Flego A, Herbert J, Waters E, Gibbs L, Swinburn B, Reynolds J, et al. Jamie's Ministry of Food: quasi-experimental evaluation of immediate and sustained impacts of a cooking skills program in Australia. PLoS One. 2014;9(12): e114673.

44. Herbert J. Jamie's Ministry of Food Victoria: participant experience evaluation. Melbourne, Victoria: Deakin University; 2015.

45. Herbert J, Flego A, Gibbs L, Waters E, Swinburn B, Reynolds J, et al. Wider impacts of a 10-week community cooking skills program - Jamie's Ministry of Food Australia. BMC Public Health. 2014;14:1161.

46. Rosenberg M. Society and the adolescent self-image. Princeton: Princeton University Press; 1965.

47. Voxco Survey Solutions. Voxco CATI@. Canada: Voxco Survey Solutions; 2018.

48. Ware J, Kosinski M, Turner-Bowker DM, Gandek B. How to score version 2 of the SF-12 $2^{\circledR}$ health survey (with a supplement documenting version 1). Lincoln: Quality Metric Incorporated; 2002. p. 29-38.

49. Bostic T, Rubio D, Hood M. A validation of the subjective vitality scale using structural equation modeling. Soc Indic Res. 2000:52:313-24.

50. Ryan R, Frederick C. On energy, personality and health: subjective vitality as a dynamic reflection of well-being. J Pers. 1997;65:529-65.

51. Tennant R, Hiller L, Fishwick R, Platt S, Joseph S, Weich S, et al. The WarwickEdinburgh mental well-being scale (WEMWBS): development and UK validation. Health Qual Life Outcomes. 2007;5:63.

52. Craig $C L$, Marshall AL, Sjostrom M, Bauman AE, Booth ML, Ainsworth BE, et al. International physical activity questionnaire: 12-country reliability and validity. Med Sci Sports Exerc. 2003;35(8):1381-95.

53. American College of Sports Medicine. The Godin Leisure-Time Exercise Questionnaire. Med Sci Sports Exercise. 1997;26(6(Suppl)):S36-8.

54. Podsiadlo D, Richardson S. The timed "up \& go": a test of basic functional mobility for frail elderly persons. J Am Geriatric Soc. 1991;39(2):142-8.

55. Massy-Westropp NM, Gill TK, Taylor AW, Bohannon RW, Hill CL. Hand grip strength: age and gender stratified normative data in a population-based study. BMC Res Notes. 2011;4:127.
56. Ireland P, Jolley D, Giles G, O'Dea K, Powles J, Rutishauser I, et al. Development of the Melbourne FFQ: a food frequency questionnaire for use in an Australian prospective study involving an ethnically diverse cohort. Asia Pac J Clin Nutr. 1994;3(1):19-31.

57. Lewis JR, Milligan G, Hunt A. NUTTAB 95 Nutrient data table for use in Australia. In: Authority NF, editor. Canberra: Commonwealth of Australia; 1995.

58. Hebden L, Kostan E, O'Leary F, Hodge A, Allman-Farinelli M. Validity and reproducibility of a food frequency questionnaire as a measure of recent dietary intake in young adults. PLoS One. 2013;8(9):e75156.

59. Petersen K, Smith J, Clifton P, Keogh J. Dietary intake in adults with type 1 and type 2 diabetes: validation of the dietary questionnaire for epidemiological studies version 2 FFQ against a 3-d weighed food record and 24-h urinalysis. Br J Nutr. 2015;114(12):2056-63.

60. Al-Delaimy WK, Slimani N, Ferrari P, Key T, Spencer E, Johansson I, et al. Plasma carotenoids as biomarkers of intake of fruits and vegetables: ecological-level correlations in the European prospective investigation into Cancer and nutrition (EPIC). Eur J Clin Nutr. 2005;59(12):1397-408.

61. Couillard C, Lemieux S, Vohl MC, Couture P, Lamarche B. Carotenoids as biomarkers of fruit and vegetable intake in men and women. $\mathrm{Br} J$ Nutr. 2016;116(7):1206-15.

62. Quiros Sauceda A, Pacheco-Ordaz R, Ayala-Zavala JF, Mendoza A, GonzalezCordova A, Vallejo-Galland B, et al. Impact of Fruit Dietary Fibres and Polyphenols on Modulation of the Human Gut Microbiota. In: Yahia E, editor. Fruit and Vegetable Phytochemicals: Chemistry and Human Health. 1. 2nd ed. Chichester: Wiley; 2018. p. 405-21. https://doi.org/10.1002/ 9781119158042

63. Williamson G. The role of polyphenols in modern nutrition. Nutr Bull. 2017; 42(3):226-35.

64. Veronese N, Solmi M, Caruso M, Giannelli G, Osella A, Evangelou E, et al. Dietary fibre and health outcomes: an umbrella review of systematic reviews and meta-analyses. Am J Clin Nutr. 2018;107:436-44.

65. Genoni A, Christophersen CT, Lo J, Coghlan M, Boyce MC, Bird AR, et al. Long-term Paleolithic diet is associated with lower resistant starch intake, different gut microbiota composition and increased serum TMAO concentrations. 2019.

66. Lockyer S, Nugent AP. Health effects of resistant starch. Nutr Bull. 2017;42(1): $10-41$.

67. Marfell-Jones M, Olds T, Stewart A, Carter L. International standards for anthropometric assessment. Rev. ed. Potchefstroom: ISAK; 2006.

68. Woodside J, Draper J, Lloyd A, McKinley M. Use of biomarkers to assess fruit and vegetable intake. Proc Nutrition Soc 2017;76(3):308-315.

69. Wang Y, Klarić L, Yu X, Thaqi K, Dong J, Novokmet M, et al. The association between glycosylation of immunoglobulin $\mathrm{G}$ and hypertension: a multiple ethnic cross-sectional study. Medicine. 2016;95(17):e3379.

70. Maes M, Kubera M, Leunis J-C. The gut-brain barrier in major depression intestinal mucosal dysfunction with an increased translocation of LPS from gram negative enterobacteria (leaky gut) plays a role in the inflammatory pathophysiology of depression. Neuroendochrinol Letters. 2008;29(1):11724.

71. O'Mahony SM, Clarke G, Borre YE, Dinan TG, Cryan JF. Serotonin, tryptophan metabolism and the brain-gut-microbiome axis. Behav Brain Res. 2015;277: 32-48.

72. Martin M. Cutadapt removes adapter sequences from high-throughput sequencing reads. EMBnet J. 2011;17(1):10-2.

73. Callahan B, McMurdie P, Rosen M, Han A, Johnson A, Holmes S. DADA2: high-resolution sample inference from Illumina amplicon data. Nat Methods. 2016;13(7):581-3.

74. Cole J, Wang Q, Fish J, Chai B, McGarrel D, Sun Y, et al. Ribosomal database project: data and tools for high throughput rRNA analysis. Nucleic Acids Res. 2014;42:D633-D42.

75. Parks H, Chuvochina M, Waite D, Rinke C, Skarshewski A, Chaumeil P-A, et al A standardized bacterial taxonomy based on genome phylogeny substantially revises the tree of life. Nat Biotechnol. 2018;36:996.

76. Mullish BH, Pechlivanis A, Barker GF, Thursz MR, Marchesi JR, McDonald JAK. Functional microbiomics: evaluation of gut microbiota-bile acid metabolism interactions in health and disease. Methods. 2018;149:49-58.

77. Hegyi P, Maleth J, Walters JR, Hofmann AF, Keely SJ. Guts and gall: bile acids in regulation of intestinal epithelial function in health and disease. Physiol Rev. 2018;98(4):1983-2023. 
78. Humbert L, Maubert MA, Wolf C, Duboc H, Mahe M, Farabos D, et al. Bile acid profiling in human biological samples: comparison of extraction procedures and application to normal and cholestatic patients. J Chromatogr B Analyt Technol Biomed Life Sci. 2012:899:135-45.

79. Franco P, Porru E, Fiori J, Gioiello A, Cerra B, Roda G, et al. Identification and quantification of oxo-bile acids in human faeces with liquid chromatography-mass spectrometry: a potent tool for human gut acidic sterolbiome studies. J Chromatogr A. 2019;1585:70-81.

80. Chan AW, Tetzlaff JM, Gotzsche PC, Altman DG, Mann H, Berlin JA, et al. SPIRIT 2013 explanation and elaboration: guidance for protocols of clinical trials. BMJ. 2013;346:e7586.

81. Chan A-W, Tetzlaff JM, Altman DG, Laupacis A, Gotzsche PC, Krleza-Jeric K, et al. SPIRIT 2013 statement: defining standard protocol items for clinical trials. Ann Intern Med. 2013;158(3):200-7.

82. Clarke G, Gorley R. PRIMER v7: User Manual/Tutorial. PRIMERv7. Plymouth: PRIMER-E; 2015.

83. Burke $V$, Beilin $\sqcup$, Dunbar DL. Family lifestyle and parental body mass index as predictors of body mass index in Australian children: a longitudinal study. Int J Obes. 2001;25:147-57.

84. Moore V, Davies M. Early life influences on later health: the role of nutrition. Asia Pac J Clin Nutr. 2001;10(2):113-7.

85. Reilly J, J. A, J., Dorosty AR, Emmett PM, Ness A, Rogers I, et al. Early life risk factors for obesity in childhood: cohort study. BMJ. 2005;330(7504):1357.

86. Birch L. Development of food preferences. Annu Rev Nutr. 1999;19(1):41-62.

87. Baird J, Fisher D, Lucas P, Kleijnen J, Roberts H, Law C. Being big or growing fast: systematic review of size and growth in infancy and later obesity. BMJ. 2005;331(7522):929.

88. Elgar FJ, Craig W, Trites SJ. Family dinners, communication, and mental health in Canadian adolescents. J Adolesc Health. 2013;52(4):433-8.

89. Tumin R, Anderson SC. Television, home-cooked meals, and family meal frequency: associations with adult obesity. J Acad Nutr Diet. 2017;117(6):937-45.

90. Sarris J, Logan AC, Akbaraly T, Amminger P, Balanzá-Martínez V, Freeman $M P$, et al. Nutritional medicine as mainstream in psychiatry. Lancet Psychiatry. 2015;2(3):271-4.

91. Peterson KE, Sorensen G, Pearson M, Hebert JR, Gottlieb BR, McCormick MC. Design of an intervention addressing multiple levels of influence on dietary and activity patterns of low-income, postpartum women. Health Educ Res. 2002;17(5):531-40.

92. Jones C, Burns S, Howat P, Jancey J, McManus A, Carter O. Playgroups as a setting for nutrition and physical activity interventions for mothers with young children: exploratory qualitative findings. Health Promot J Austr. 2010;21(2):92-8.

93. Brown PR, Brown WJ, Miller YD, Hansen V. Perceived constraints and social support for active leisure among mothers with Young children. Leisure Sciences. 2001;23(3):131-4

94. Andajani-Sutjahjo S, Ball K, Warren N, Inglis V, Crawford NT, 15. Perceived personal, social and environmental barriers to weight maintenance among young women: A community survey. J Behav Nutr Phys Act. 2004;1(1):15.

95. Pollard CM, Miller MR, Daly AM, Crouchley KE, O'Donoghue KJ, Lang AJ, et al. Increasing fruit and vegetable consumption: success of the Western Australian go for $2 \& 5$ campaign. Public Health Nutr. 2008;11(3):314-20.

96. Gazzaniga FS, Kasper DL. Veggies and intact grains a day keep the pathogens away. Cell. 2016;167(5):1161-2.

97. Holscher HD. Dietary fiber and prebiotics and the gastrointestinal microbiota. Gut Microbes. 2017:8(2):172-84.

98. Diether NE, Willing BP. Microbial Fermentation of Dietary Protein: An Important Factor in Diet - Microbe - Host Interaction. Microorganisms. 2019;7(1):19. https://doi.org/10.3390/microorganisms7010019.

99. Molinero N, Ruiz L, Sanchez B, Margolles A, Delgado S. Intestinal Bacteria interplay with bile and cholesterol metabolism: implications on host physiology. Front Physiol. 2019;10:185.

100. Prochaska J, Velicer W, Rossi J, Goldstein M, Marcus B, Rakowski W. Stages of change and decisional balance for twelve problem behaviours. Health Psychol. 1994;13:39-46.

101. Herbert J, Flego A, Gibbs L, Young D, Nichols L, Waters E, et al. Jamie's Ministry of Food Victoria: participant experience evaluation. Melbourne: Deakin University; 2015.

102. Ware J Jr, Sherbourne CD. The MOS 36-item short-form health survey (SF-36): I. conceptual framework and item selection. Med Care. 1992;30(6):473-83.

\section{Publisher's Note}

Springer Nature remains neutral with regard to jurisdictional claims in published maps and institutional affiliations.
Ready to submit your research? Choose BMC and benefit from:

- fast, convenient online submission

- thorough peer review by experienced researchers in your field

- rapid publication on acceptance

- support for research data, including large and complex data types

- gold Open Access which fosters wider collaboration and increased citations

- maximum visibility for your research: over $100 \mathrm{M}$ website views per year

At BMC, research is always in progress.

Learn more biomedcentral.com/submissions 